Stoa

Vol. 3, no. 5, 2012, pp. 159-179

ISSN 2007-1868

\title{
POBLACIONES DE MODELOS Y DINÁMICAS CIENTÍFICAS
}

\author{
Mario Casanueva \\ Departamento de Humanidades \\ Universidad Autónoma \\ Metropolitana-Cuajimalpa. \\ casa@xanum.uam.mx \\ Diego Carlos Méndez \\ Departamento de Ciencias \\ de la Comunicación \\ Universidad Autónoma \\ Metropolitana-Cuajimalpa \\ dmendez@correo.cua.uam.mx
}

RESUMEN: El trabajo contribuye al desarrollo de una epistemología evolucionista de teorías científicas, desde una perspectiva cercana a la del Programa Estructuralista. Introduce herramientas metateóricas diferentes a las que propone el Programa, pero que guardan afinidades estrechas con las del estructuralismo. Se argumenta a favor de reconstruir modelos teóricos como grafos representacionales en lugar de predicados conjuntistas. El trabajo introduce los términos "población de modelos" y "dominio temático", y pormenoriza las semejanzas y diferencias que guardan, respectivamente, con las nociones estructuralistas de red teórica y holón teórico. También se explora la idea de nicho conceptual, en tanto región análoga en las estructuras de los modelos que componen una población. Finalmente, el texto discute la pertinencia del aparato propuesto para dar cuenta de los mecanismos y patrones de la evolución teórica en ciencia.

PALABRAS CLAVE: grafo representacional $\cdot$ población de modelos $\cdot$ dominio temático $\cdot$ nicho de concepto $\cdot$ evolución teórica

SUMMARY: This paper contributes to the development of an evolutionary epistemology of scientific theories, from a vantage point closely related to the Structuralist Program. The authors introduce a metatheoretical tool kit which differs from that of the Program, but nonetheless has a close affinity with structuralism's resources. The argument is made in favor of reconstructing theoretical models by means of representational graphs instead of set-predicates. 
The terms "model population" and "thematic domain" are introduced, and a detailed account of their differences and similarities with the respective structuralist notions of theory net and theory holon is given. The idea of a conceptual niche as an analogous region in the structures of models that compose a population is also explored. The paper closes with a discussion of the proposed apparatus' fruitfulness in revealing and accounting for mechanisms and patterns of theory evolution in science.

KEYWORDS: representational graph - model-population - thematic domain concept-niche $\cdot$ theory evolution

\section{Tipos y poblaciones}

La dinámica e interacción de las teorías puede ser representada sobre la base de analogías de la evolución orgánica que enfaticen las variaciones estructurales de los modelos teóricos. Es probable que cualquier intento de este tipo se enfrente a problemas similares a los que se enfrentó la biología en el modo de mirar a sus objetos. En particular el problema de la existencia de clases naturales de organismos y la cuestión subsecuente en torno a los criterios de pertenencia a dichas clases. La taxonomía linneana plantea que las clases son pequeñas e irrelevantes desviaciones de una esencia; por el contrario, el enfoque poblacional enfatiza las variaciones reales entre poblaciones de organismos, que conforman una red de descendencia: una visión se centra en el tipo ( $\varepsilon เ \delta \circ \varsigma)$; la otra en la población. Se trata de enfoques opuestos que no se limitan al campo de la biología por lo que, necesariamente, cualquier intento de construcción de una epistemología evolucionista de teorías se verá sometido a las tensiones entre estos enfoques. Por esto, conviene exponer sus diferencias.

El enfoque esencialista describe a los individuos en términos de características esenciales y accesorias, y exige que todos los miembros de una clase compartan las primeras, mismas que están fijadas de antemano; por el contrario, el enfoque poblacional no establece semejante distinción, pues otorga a todas las características la misma importancia taxonómica, requiere del reconocimiento de las diferencias reales entre los organismos, sin prejuzgar cuáles son exactamente los aspectos que deben ser compartidos. Dado que cada individuo es único, no basta recuperar las características esenciales que darían un individuo tipo, sino que es necesario considerar todas las presentes en los individuos 
de la población. Por otra parte, el enfoque poblacional suministra los criterios de pertenencia al grupo en términos de relaciones de interfecundidad con los demás integrantes del grupo. ${ }^{1}$

En la biología es común un uso ambiguo de los conceptos de especie y población, mismos que, sin serlo, en ocasiones se consideran sinónimos. Una diferencia clara entre ambos es que las poblaciones son grupos interfecundos de organismos y una propiedad inherente a ellas es poseer un referente espacio-temporal. Esto último no necesariamente se aplica al término 'especie', si bien se puede considerar a la especie como la suma de las poblaciones que la componen, dándole en consecuencia una ubicación espacio-temporal. También es posible reconstruir el concepto con base en ejemplares aislados, como sucede en la paleontología. Cualquier analogía evolucionista sobre la evolución cultural o científica debe considerar esta diferencia.

\section{Un enfoque poblacional para el estructuralismo}

Aunque el aparato analítico de la concepción estructuralista ofrece herramientas para desarrollar un enfoque poblacional metacientífico, hasta ahora sus interpretaciones son esencialistas. ${ }^{2}$ Este aspecto se manifiesta, por ejemplo, en la distinción entre ley fundamental y leyes especiales, la cual supone la existencia de una esencia compartida por todos los modelos de una red teórica, y de una parte accesoria mutable. El esencialismo dentro de la concepción estructuralista se pone de relieve al plantear la pertenencia a una teoría en términos sustantivistas, es decir, en términos de particulares condiciones estructurales, necesarias y suficientes para que un modelo pertenezca a una teoría determinada. En nuestra opinión, esta manera de ver las cosas soslaya la importancia real de las variaciones reales de los modelos y, en consecuencia, es inadecuada para el planteamiento de una dinámica teórica.

La adquisición de un enfoque poblacional, si bien constituye un cambio dentro del estructuralismo, es mucho menos que una "revolución", pues mantiene las intuiciones respecto a la dimensión conceptual que subyacen al formalismo estructuralista, al tiempo que lo

${ }^{1}$ Cfr. v.g., Lewontin 1975, p. 5 y la introducción de Mayr 1942.

${ }^{2}$ Al menos ésta es la nota de Balzer, Moulines y Sneed 1987 y de Díez y Moulines 1999, caps. 10 y 13 
dota de herramientas que incrementan su capacidad de competencia y diálogo con otras corrientes de la filosofía de la ciencia. A grandes rasgos, nuestras propuestas son:

i. Presentar modelos mediante grafos representacionales (con extensas notas a pie), cuyos componentes se resumen en el recuadro a continuación. En este texto, en ocasiones los términos "grafo" y "modelo" se emplean casi como sinónimos, permitiendo que el contexto diferencie entre ambos.

\begin{tabular}{|c|l|l|}
\hline Componente & Puntos & Flechas \\
\hline Conceptual & $\begin{array}{l}\text { Sistemas o entida- } \\
\text { des (conjuntos y } \\
\text { conjuntos escalón) }\end{array}$ & $\begin{array}{l}\text { Funciones, defini- } \\
\text { ciones funcionales } \\
\text { e identificaciones. }\end{array}$ \\
\hline
\end{tabular}

ii. Cambiar el énfasis actual sobre los conceptos de red teórica y holón teórico en favor de los conceptos de población de modelos y dominio temático.

iii. Incluir una representación espacial de modelos, poblaciones de modelos y dominios temáticos que enfatice sus variaciones estructurales.

\subsection{Grafos}

La sustitución de los predicados conjuntistas por grafos no presenta problemas en principio, pues los primeros se pueden traducir a los segundos y viceversa. La presentación a modo de grafo permite apreciar, literalmente de un vistazo y de manera completa, la estructura conceptual de un modelo y en consecuencia facilita la comparación entre ellos. Aunado a esto, revela ciertos aspectos metacientíficos no tan fácilmente visibles en los predicados conjuntistas, en particular las rutas de inferencia que ofrece el modelo en cuestión, lo que posibilita identificar en dónde radica su capacidad explicativa.

Si los dominios de una estructura se representan como puntos y las relaciones por flechas, a cada tipo de modelo de una teoría se le puede asociar un grafo; éstos permiten diferentes niveles de profundidad en el análisis. Cada punto o flecha puede en el fondo ser visto como un sistema. Esto es, como una estructura susceptible de ser desplegada o analizada más a fondo e, inversamente, determinadas subestructuras 
de un grafo se pueden colapsar en un punto o una flecha según el caso; de este modo, los puntos pueden representar subestructuras o grafos completos. A título de ejemplo, las dos siguientes figuras muestran esta idea de diferentes niveles de análisis para una teoría concreta, la teoría de la fecundación unigamática. ${ }^{3}$
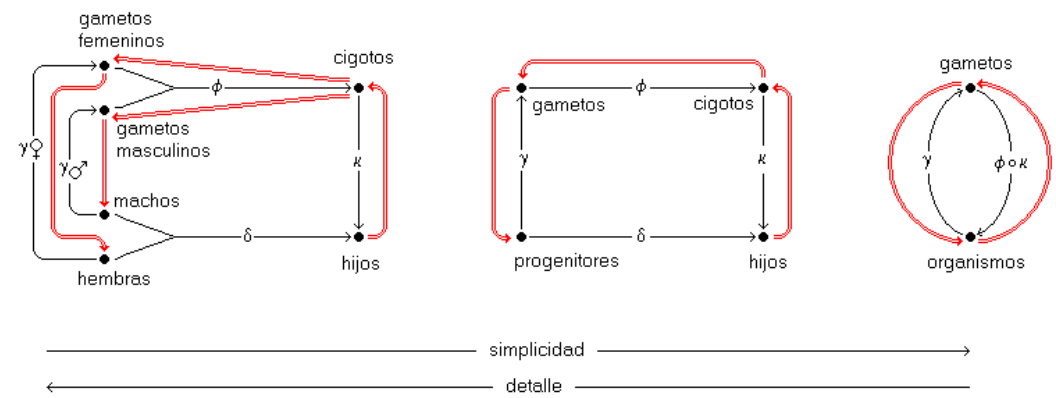

Figura 1. Estructura conceptual de la Teoría Unigamética de la Fecundación vista a diferentes niveles de profundidad. La interpretación de las flechas es: $\delta$ descendencia, $\gamma q$ gametogénesis femenina, $\gamma_{\sigma}{ }^{7}$ gametogénesis masculina, $\phi$ fecundación, $k$ crecimiento y y gametogénesis (general). La ley fundamental se indica con flechas dobles

Figura 1: Estructura conceptual de la teoría unigamética.

Por supuesto que estos diferentes niveles de análisis también pueden introducirse en una reconstrucción conjuntista clásica pero este aspecto, hasta donde sabemos, no había sido señalado.

En los grafos pueden identificarse las formas en que (desde un punto de vista epistemológico) funcionan las diferentes partes o conjuntos de partes que conforman un modelo; entre otras:

(i) Las flechan enlazan origen (orígenes) y destino, y en general pueden ser vistas como reglas de inferencia que permiten obtener datos acerca del último a partir de datos acerca del (de los) primero(s). La concatenación (composición) de varias flechas genera rutas de inferencia. Cabe aclarar que pueden existir flechas con dos o más orígenes, pero no con dos o más destinos; tanto origen (orígenes) como destino pueden ser puntos o flechas.

\footnotetext{
${ }^{3}$ Una reconstrucción formal de la red de la fecundación unigamática puede verse en Casanueva 2003, cap. 2
} 


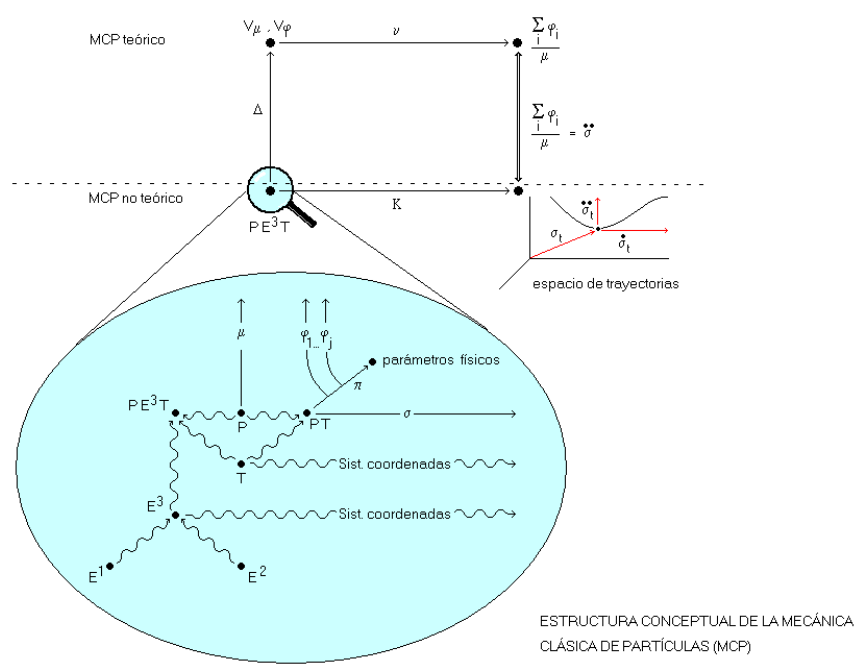

Figura 2: Estructura conceptual de la mecánica de partículas clásica.

(ii) Las leyes (especiales y particulares) se representan mediante distintos tipos de flechas, o bien, con flechas de colores distintos. Las condiciones de ligadura y las relaciones interteóricas son flechas que engarzan distintos grafos. Un funcional es una flecha que engarza flechas.

(iii) Las flechas onduladas representan estructuraciones. Éstas son un tipo particular de funciones que operan sobre las entidades origen y permiten obtener: múltiples arreglos (combinaciones, agrupaciones, secuencias, agrupaciones de agrupaciones, agrupaciones de secuencias, secuencias de secuencias, combinaciones de secuencias de agrupaciones, etc.) que forman "nuevas" entidades, a saber: las entidades destino. Desde un punto de vista puramente sintáctico, las estructuraciones pueden conceptuarse como definiciones, pues no añaden novedades ontológicas y en principio son eliminables y no creativas pero, desde un punto de vista semántico, éstas más bien corresponden a postulados de existencia de las entidades destino, pues no son sino el resultado de aplicar diversos operadores 
conjuntistas sobre los términos primitivos. ${ }^{4}$ No cualquier combinación construible de entidades es aceptable por la teoría como algo que posee referente. ${ }^{5}$

(iv) En los grafos se diferencian los conceptos o subestructuras que conforman la base (de datos) contra la que se contrasta un modelo (BD) de aquellos conceptos cuyo uso presupone la validez de las leyes propuestas (y en consecuencia no deben ser usados para contrastar la teoría). Estos últimos conforman la superestructura teórica del modelo (SET). Mutatis mutandis, BD y SET "corresponden" respectivamente a lo que en jerga estructuralista se denomina $M_{p p}$ y "conjunto de términos $T$-teóricos" (la correspondencia no es exacta, la caracterización de BD y SET no se limita a los términos primitivos, incluyen las estructuraciones y sus entidades destino). Aquí esta demarcación se establece mediante una línea punteada que divide al grafo en dos regiones.

(v) Subestructuras: Los grafos (o las estructuras conceptuales a las que representan) pueden conceptualizarse como mosaicos de partes. Dentro de cada grafo se pueden distinguir diferentes subestructuras, subgrafos o "recortes". BD y SET no son sino dos muy importantes subgrafos, pero otro tipo de subestructuras también pueden revelarse como importantes al análisis. Los elementos individuales (puntos y flechas) constituyen los recortes o subestructuras más simples, pero pueden componerse o diferenciarse subgrafos que incluyan más de un elemento individual. Los elementos que constituyen una subestructura deben enlazarse de tal manera que entre ellos exista continuidad. La idea es que la unión de partes individuales estructuralmente ligadas genera partes más grandes (tal conexión no toma en cuenta la dirección de las flechas).

\footnotetext{
${ }^{4}$ En este sentido nuestro trabajo sigue las líneas generales de Poincaré 1908, p. 104.

${ }^{5}$ Por ejemplo, los genotipos no son sino determinadas combinaciones de genes y constituyen uno de los dominios centrales de diversas genéticas, pero las ternas conformadas por individuos de ojos azules de la generación 8 , individuos que poseen lóbulos separados de la generación 32 e individuos hemafroditas de las 452 y 5015 -aunque sea construible con los términos primitivos de la genética- es una entidad absurda que no aparece en ninguna de sus leyes.
} 


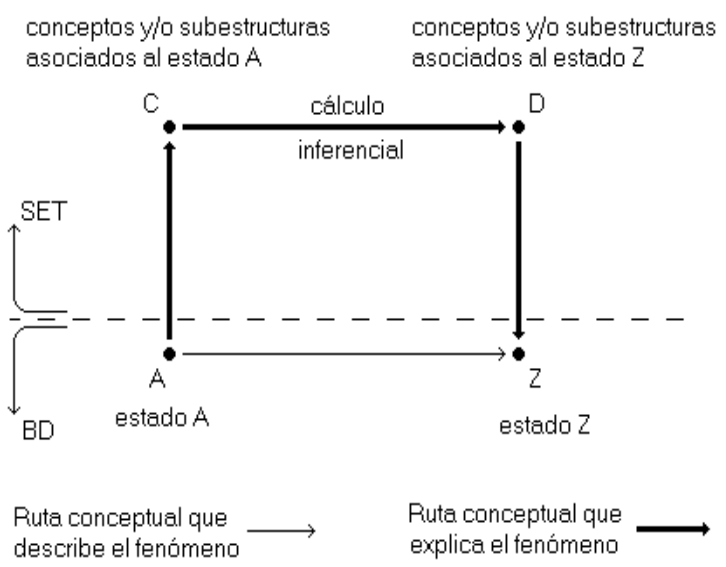

Figura 3: Diagrama de rutas conceptuales.

Un modelo es algo que se crea para responder una pregunta; ésta puede ser representada de manera muy general mediante: " $A \rightarrow Z$ ", donde la flecha indica un proceso o la adscripción de la propiedad $B$ a los objetos que constituyen $A$. Como se ha dicho, la flecha es una regla de inferencia (o un conjunto de ellas) que permite obtener ciertos datos acerca del destino a partir de datos acerca del (los) origen(es). ${ }^{6} \mathrm{El}$ fenómeno $A \rightarrow Z$ se encuentra en la porción correspondiente a la base de datos. Para explicarlo, el modelo postula una serie de entidades (puntos: $C, D$ ) y relaciones (flechas) que constituyen una ruta alternativa que igualmente engarza $A$ con $Z$, pasando, si la hay, por la región de la superestructura teórica. Las dos rutas son puestas en relación, de tal suerte que se conforma un circuito en el que radica la capacidad explicativa del modelo. ${ }^{7}$ Ambas juegan, de alguna manera, el papel que el modelo de explicación que Hempel les asignaba a explicans y explicandum, correspondiendo a " $A \rightarrow Z$ " el último. Si las rutas se relacionan mediante la igualdad, estaremos en presencia de un circuito conmutativo, pero pueden darse otros tipos de relación entre las rutas

\footnotetext{
${ }^{6}$ El papel de la matemática y la lógica no sería otro que el de construir nuevas rutas inferenciales, postulando definiciones adicionales que constituirían nuevos puntos, y relaciones inferenciales, que formarían nuevas flechas.

${ }^{7}$ Estas ideas son similares a las desarrolladas por Barwise y Seligman 1997.
} 
(inclusión, desigualdad, diferencia). Lo importante es que esta relación nos permita inferir algo de una a partir de la otra. La forma en que se relacionan caracteriza de alguna manera el grado de suficiencia empírica del modelo; la conmutatividad sería la mayor suficiencia, pues es la que otorga más información.

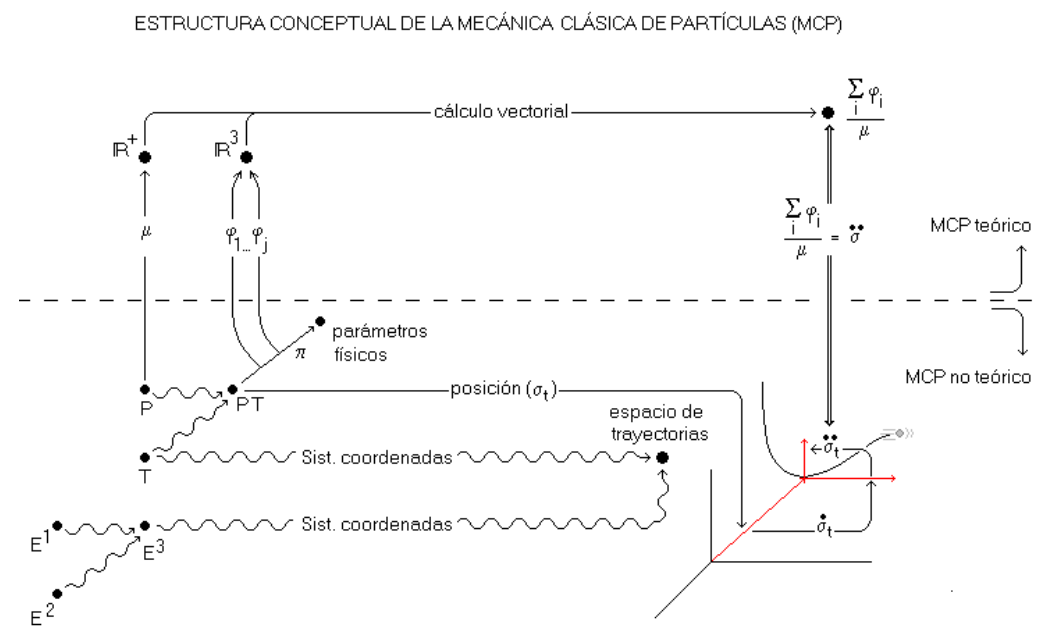

Figura 4: Estructura conceptual de la mecánica clásica de partículas.

Para fijar ideas, a continuación presentamos como funcionan estas rutas en la mecánica clásica de partículas (MCP).

El problema para el cual la MCP pretende ser una respuesta es: cómo ocurre el movimiento de las partículas en el espacio-tiempo. El momento $A$ está representado por las nociones de partícula, tiempo y espacio, el estado $Z$ constituiría una descripción del movimiento en términos de posiciones, velocidades y aceleraciones instantáneas (flechas rojas). La relación existente entre $A$ y $Z$ es justamente aquella que permite la construcción de un sistema de coordenadas y la introducción de posiciones, velocidades y aceleraciones como entidades vectoriales dentro de tal sistema. De esta manera $A \rightarrow Z$ representa la pregunta central de MCP.

El punto $B$ estaría representado por los valores masa (reales positivos) y los valores fuerza (vectores en $\mathbb{R}^{3}$ ). La flecha entre $A$ y $B$ vendría dada por la función masa y los diferentes funcionales fuerza que de- 
penden de la asignación de determinados parámetros físicos a cada partícula en cada instante (II). El punto $C$ está representado por el vector que indica la sumatoria de las fuerzas multiplicada por el inverso de la masa. Así, la flecha $B \rightarrow C$ representa el cálculo vectorial que nos indica cómo sumar vectores y cómo multiplicarlos por escalares. Por último, la flecha $C \rightarrow Z$ ilustra la segunda ley de Newton que iguala $C$ a la aceleración instantánea de la partícula postulada en $Z$.

\subsection{Poblaciones}

Los modelos pueden describirse como mosaicos de las subestructuras que los componen, así $m=\left\langle S E_{1}, \ldots, S E_{n}\right\rangle$, donde cada $S E_{i}(1 \leq i \leq n)$ es una subestructura, y cualesquiera dos subestructuras distintas son ajenas entre sí. Si se considera la totalidad de subestructuras presentes en una población y se atiende a la "función" que éstas desempeñan en los modelos en que ocurren -por ejemplo, en MCP, las distintas especializaciones del concepto de fuerza podrían verse como elementos de una misma clase de equivalencia- es posible generar una "partición" sobre el conjunto total de subestructuras. De esta manera, los modelos de la población se pueden obtener como diferentes recombinaciones de los elementos de las distintas "clases de equivalencia". 8

La red teórica constituye un caso límite de una población. Sus modelos están estructurados por la relación de especialización, todos comparten la misma ley fundamental. ${ }^{9}$ En contrario, una población es un conjunto de modelos que guardan entre sí una relación de semejanza conceptual, estadísticamente significativa. Los modelos de una red comparten determinados puntos y flechas concretas, pero para pertenecer a una población no se exige esto; sí se exige que se comparta

\footnotetext{
${ }^{8}$ En estricto sentido, no se trata de una partición, pues las clases que la conforman en ocasiones pueden no ser ajenas entre sí, pero en este caso el elemento compartido es sólo uno y siempre el mismo, a saber: el conjunto vacío o el cero. Por eso hemos considerado que representan una cuasi partición o "partición". Este enfoque tiene la ventaja de permitir la introducción de matrices.

${ }^{9}$ Como hemos mencionado, ya que la pertenencia a la red está dada por la posesión de determinados puntos y flechas en el grafo que representa sus modelos, la caracterización de las redes puede calificarse de esencialista. Tal propiedad también se observa en la importancia de segundo orden otorgada a aquellos términos que se pueden introducir como definiciones (estructuraciones) a partir de los primitivos. Por otra parte, la aserción empírica central de una teoría $T$ se postula en términos de la subsunción de modelos potenciales parciales de $T$ bajo su porción $T$-teórica pero, aún cuando se aclara que tal subsunción es aproximada, se resta importancia al hecho de que las teorías se modifican al enfrentarse a sus competidores.
} 
algo, pero no se exige que ese algo esté previamente determinado, la diferencia puede estar incluso en aquellos puntos y flechas que antes se consideraban esenciales.

Para el enfoque tipológico, la esencia de una teoría está fijada en el núcleo sin especializaciones. Pero muchas veces tal núcleo sólo aparece, si acaso, en un contexto educativo propio de los libros de texto, y en consecuencia es poco representativo de la historia real de la teoría, lo cual lo hace susceptible a la crítica de constituirse en un artefacto que utiliza el reconstructor. A pesar de estas diferencias existe una clara semejanza entre los conceptos de red teórica y población de modelos: ambos son entidades genidénticas (susceptibles de modificación sin pérdida de identidad).

Para el estructuralismo una teoría es una red diacrónica; para el enfoque que proponemos es una población diacrónica. La diferencia estriba en que una población puede incluir dos o más redes distintas (su reconstrucción implica cambios en los $M_{p p}$ y la ley fundamental), aunque muy semejantes. Para identificar una red basta con identificar al núcleo (sin especializaciones); para identificar una población es menester identificar un grupo estadísticamente representativo de la misma.

En dos modelos distintos de una misma población, pero de diferentes redes, las leyes están formuladas de tal manera que conservan la misma estructura, pero cabe la posibilidad de que sus términos sean interpretados de manera distinta (aunque podríamos decir que la interpretación es semejante pues, si se considera la totalidad de las subestructuras presentes en los modelos, notaremos que existe una fuerte intersección). Por lo general, aunque una población puede incluir más de una red, entre sus integrantes existe una relación de traducibilidad punto por punto.

La semejanza constituye un indicador del tamaño relativo de la intersección de dos grafos, el cual puede capturarse por muchos indices. El que se elija debe justificarse en cada caso concreto. De cualquier manera, el alto grado de semejanza entre modelos es lo que delimita una población; dos modelos que acusen una valor de semejanza por arriba de una cierta cota pertenecen a una población. Dicho umbral es un concepto difuso, cuyos límites están en discusión y dependen de contextos específicos, pero igual sucede con otros conceptos -por 
ejemplo el de comunidad científica. No obstante, el concepto de población posee una cierta robustez pues, si se abandona el terreno estrictamente conceptual, veremos que dos modelos de redes distintas, pero de una misma población, comparten los mismos procedimientos empíricos para la identificación de entidades y la determinación de funciones. ${ }^{10}$

\subsection{Dominios temáticos}

Empleamos la expresión 'dominio temático' en el mismo sentido en el que se usan expresiones tales como: 'el dominio de la herencia', 'el dominio de las teorías de la evolución', 'el dominio de las teorías de la luz', etcétera; e incluso, si la metodología que pretendemos desarrollar resulta adecuada para ello, el 'dominio de las cosmovisiones' o cualquier otro territorio extracientífico (magia, religión, mitología). Un dominio temático es el espacio conceptual donde ocurren las diferentes propuestas alternativas a la pregunta basal para la cual los modelos pretenden ser una respuesta. Las diferentes mecánicas pretenden resolver el problema del movimiento; las diferentes genéticas el de la trasmisión de características; las diferentes teorías evolutivas, el del mecanismo de la evolución, etcétera. Y esta pretensión común es lo que les confiere la posibilidad de diálogo y/o conflicto. Un dominio, en tanto pregunta, preexiste a los modelos que se insertan en él (en tanto respuestas), modificándolo. Sin embargo, tales modelos pueden no compartir la caracterización o la importancia relativa otorgada al problema. Como se infiere, un dominio puede abarcar poblaciones distintas. En dos modelos de un mismo dominio, pero de diferentes poblaciones, las leyes pueden presentarse si acaso mediante estructuras análogas. Como veremos más adelante, si tales estructuras pertenecen al mismo nicho, podría postularse la existencia de una relación entre ellas.

A nuestro juicio la introducción del concepto de dominio enriquece considerablemente las posibilidades de inteligibilidad de la dinámica de los modelos y sus poblaciones. Al ser el espacio de confluencia en

\footnotetext{
${ }^{10}$ En ocasiones los modelos de una determinada población son postulados sólo como posibilidades teóricas, sin llegar a desarrollar métodos empíricos de identificación y determinación; sin embargo, dan por buenos los que están asociados a otro u otros modelos de ésta, para los cuales se cumple la restricción antes mencionada.
} 
donde "discuten" los diferentes modelos sobre un tema, es lógico pensar que entre ellos se darán influencias o contraposiciones que resulten en su modificación, particularmente en la "incorporación" de estructuras de uno en el otro, o en la "imitación" de estructuras de uno por parte del otro. El dominio como espacio abstracto refleja las comunicaciones reales entre científicos de un campo específico. La pertinencia particular del concepto queda clara en el siguiente ejemplo: si no consideramos que las escuelas biometrista y mendeliana pertenecían a un mismo dominio cuyos miembros "dialogaban", es difícil entender por qué los mendelianos incorporaron la tabla de correlación. ${ }^{11}$

Así como redes y poblaciones son entidades genidénticas, también lo son dominio temático y holón teórico; sin embargo, existen distinciones entre ambos. Las principales son:

(1) Un dominio temático está acotado respecto a un problema o tema. Entre dos grafos de poblaciones distintas de un mismo dominio puede no existir ninguna de las relaciones interteóricas actualmente privilegiadas por el estructuralismo; a diferencia de esto, el holón está pautada por las distintas relaciones interteóricas. Ningún holón contempla modelo alguno que no posea al menos una relación interteórica con la teoría central, además, un holón puede incluir cualquier relación interteórica con cualquier otra teoría que no necesariamente está incluida en el mismo dominio.

(2) A diferencia de un holón, un dominio induce una representación espacial de las poblaciones que se pueden plasmar en él, misma que destaca las relaciones de semejanza entre modelos. Si cada grafo corresponde a una determinada familia de estructuras $\left\langle S E_{1}, \ldots, S E_{n}\right\rangle$, es posible considerar que tales tuplas representan vectores de un espacio $n$-dimensional y, en consecuencia, es posible asignarles puntos en ese espacio. De esta manera, cabe representar las poblaciones de grafos como nubes de puntos, cuya conformación espacial pone de relieve

\footnotetext{
${ }^{11}$ Dicho sea de paso, el concepto de dominio es, en algún sentido, antikuhniano, pues al contemplar la totalidad de variantes en discusión, y otorgarles en principio la misma importancia, no se corresponde con la idea de un paradigma hegemónico. La importancia de los biometristas no es cabalmente recuperada bajo la óptica del desarrollo de la "ciencia normal" que pretende una visión de la labor de los científicos reducida a un "crear más de lo mismo". Poco hay en Mendel que sea "más de lo mismo" respecto al desarrollo de un enfoque poblacional en genética. Este enfoque fue desarrollado y dirigido por miembros de la escuela biometristas (misma que en un principio se vio más como una alternativa a Mendel que como un complemento de éste).
} 
sus principales ejes de variación estructural y permite detectar tanto los subcúmulos conformados por modelos que guardan una mayor similitud entre sí como el grado de diferencia entre puntos de distintos subcúmulos. Así, en un dominio se pueden establecer índices de semejanza intermodélica a partir de la ubicación de los modelos respecto a determinados ejes de variación conceptual. ${ }^{12}$ Así, la idea de dominio temático redimensiona la relación de semejanza entre modelos. Por su parte, un holón simplemente demarca y tipifica las relaciones interteóricas de una determinada red.

Para aclarar ideas, a continuación mostramos una representación muy sencilla del dominio de las teorías de la herencia biológica, esgrimidas durante las últimas dos décadas del siglo xix y primera del siglo xx. Aquí, entre otras, figurarían la teoría del germoplasma de Weismann (1893), la ley de herencia ancestral de Galton (1889), las modificaciones que Pearson y otros biometristas realizaron a ésta última, la teoría de Mendel (que si bien data de 1865, no impactó la comunidad científica hasta 1900) y la reinterpretación que Bateson (1902) hace a ésta, la teoría cromosómica de la herencia mendeliana propuesta inicialmente por Sutton (1903), la pangénesis celular de De Vries (1910) y varias otras más.

Todas estas teorías suponen una idea de descendencia y destacan alguna forma de describir a los individuos como una combinación de características heredables. Casi todas plantean una determinada forma de reproducción, algunas hacen hincapié en la naturaleza celular de las características que describen a un individuo, la mayoría postulan ciertas entidades (materiales o no) de la herencia, cuyo desenvolvimiento, despliegue, acción a distancia u otro tipo de relación causal genera las características heredables, y algunas más postulan entidades matemáticas que sirven para predecir la hechura de un individuo si se conoce las constituciones de sus ancestros.

En el siglo xx el cúmulo que abarca a los rótulos Darbshire, Galton, Pearson ( 1 y 2) y Warren corresponde a los grafos de las propuestas elaboradas por personajes de la llamada escuela biometrista, que tuvo

\footnotetext{
${ }^{12}$ En nuestra opinión, una diferencia fundamental entre dominio y holón radica en que el primero no sólo establece relaciones entre modelos sino que ubica las posiciones relativas de éstos en un espacio de posibilidades no necesariamente concretizadas. Esta concepción abre la puerta a la discusión de conceptos tales como atractor, direccionalidad, pronósticos de desarrollo de nuevos modelos, etcétera.
} 
su auge en la Inglaterra de este periodo. El grupo formado por Weis 1 y Weis 2 indica dos modalidades de la teoría de la continuación del germoplasma de Weismann. Sutton y Determinamental corresponden a dos teorías cromosómicas de la herencia que incorporan elementos mendelianos. El grupo que incluye los puntos Mendel y Bateson corresponde a la teoría de Mendel y a la reinterpretación que hizo Bateson de ésta. Por último, Yule es un punto aislado y pone de relieve una formulación que recuperara la ley de herencia ancestral de los biometristas en términos cuasi mendelianos.

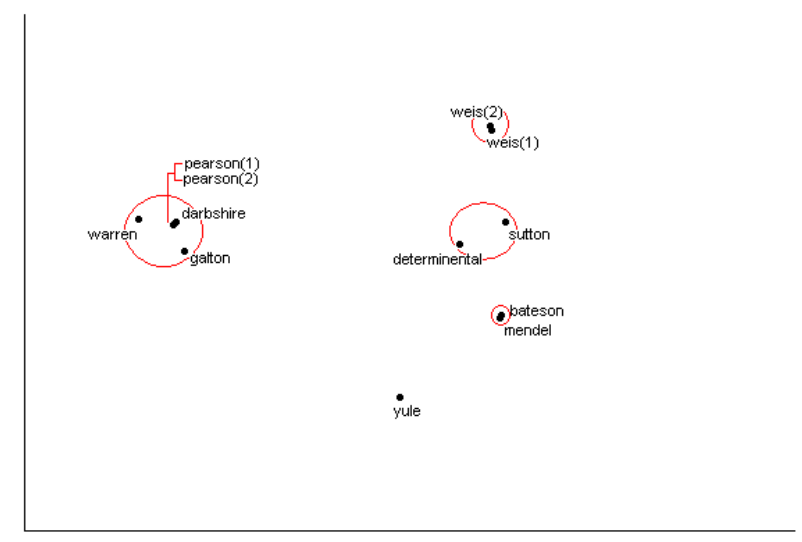

Figura 5: Tabla de distancias interteóricas.

La figura se obtuvo a partir de una tabla de distancias entre las teorías en cuestión, la cual se sometió a una escalación multidimensional con el programa ASCAL para así dar una representación espacial en dos dimensiones. Aquí el índice de semejanza interteórica fue distancia euclidea. Como puede verse, el diagrama recupera las intuiciones historiográficas del caso.

Por una mera cuestión de facilidad de obtención, el ejemplo anterior se inserta en un marco geométrico. Actualmente consideramos que es más adecuado realizar tales comparaciones entre poblaciones de un mismo dominio temático bajo un enfoque topológico. La comparación geométrica resulta adecuada entre modelos de una misma población, pero para comparar modelos de poblaciones distintas de un mismo dominio es preferible un enfoque topológico. Esto es así por 
dos cuestiones que quizá no sean enteramente independientes: (1) nada nos garantiza que el espacio conceptual que conforma un dominio sea, en efecto, un espacio métrico y (2) en estricto sentido, una comparación geométrica exige la preservación del significado que se presenta sólo en las distintas especializaciones de una red, o si acaso, en las de una misma población.

En las poblaciones distintas de un dominio por lo general existen distintas analogías entre subestructuras de modelos de poblaciones distintas; por ejemplo, la entidad "gen" de los modelos que conforman la red de la herencia mendeliana es análoga a la entidad "gémula" de los modelos que conforman la red de la herencia con base en la pangénesis, ${ }^{13}$ aún cuando los significados de "gen" y "gémula" sean distintos. Los primeros son factores causales de las características, mientras que las gémulas dan pie a células, y éstas se estructuran en características. Los significados son distintos, pero su "función" (el tipo de engarce con las demás entidades y relaciones de los modelos) es análoga, pues ambos son factores hereditarios que se trasmiten de una generación a otra. Dichas relaciones analógicas ponen de relieve que las semejanzas entre modelos de un dominio no necesariamente son proximidades métricas y, en consecuencia, no es menester que el espacio conceptual en el cual se ubican los modelos sea geométrico. Sin embargo, es posible construir un espacio topológico basado en una analogía, la cual no se puede recuperar mediante un morfismo, peso sí con algún tipo de mapeo entre estructuras. Esto tiene implicaciones en el tipo de semejanzas susceptibles de análisis, pues existen índices de semejanza que no son métricos.

\subsection{Nichos}

Respecto a los problemas asociados al llamado cambio semántico, actualmente estamos trabajando la idea de "nicho conceptual" pues, en nuestra opinión permitiría una comparación a nivel "meta" que eludiría los problemas del cambio semántico. Ya no se compara la presencia o ausencia de la misma subestructura, sino de estructuras del mismo tipo. ${ }^{14}$

\footnotetext{
${ }^{13}$ Darwin 1883.

${ }^{14}$ Arriba se señaló que los modelos se pueden obtener como recombinaciones de los elementos de las distintas clases de equivalencia de una partición sobre la totalidad de subes-
} 
Para aclarar ideas: los términos gen (mendelismo), pangen (De Vries), gémula (Darwin), elemento (Mendel), bióforo (Weismann), unidad fisiológica (Spencer), etc., pueden verse como subestructuras que comparten el mismo nicho, pues todos se refieren a factores causales de la herencia, pero no son caracterizados de igual manera, ni establecen las mismas relaciones en los modelos en los cuales aparecen. Las teorías de la reproducción sexual o asexual pueden ser instanciaciones del nicho "reproducción", pues ambas sirven para la construcción de la base de contrastación en distintas teorías de la herencia. Incluso, no parece descabellado asimilar al mismo nicho los conceptos de "masa" y "extensión" en teorías mecánicas, o "selección natural" y "tendencia a la perfección" en teorías evolutivas.

Un nicho es, pues, una región de variación donde las subestructuras que lo pueden ocupar "funcionan" (desde un punto de vista epistemológico) de la misma manera; esto es, pretenden enlazar el mismo tipo de conceptos. Las distintas instanciaciones de un mismo nicho no interrumpen la conmutatividad del circuito explicativo. Dicho de otro modo, dos subestructuras del mismo nicho son intercambiables, siempre y cuando se cambie la interpretación de las entidades. No sólo existen cambios aislados de subestructuras, sino que cabe la posibilidad de que salvaguardar la coherencia del grafo implique que una determinada instanciación deba necesariamente acompañarse de otra $\mathrm{u}$ otras, en otras regiones (existen cambios ligados). Las estructuras de un mismo nicho son polos de una relación determinada por las reglas de "traducción" que expresan las analogías entre los distintos grafos.

\section{Notas para una dinámica científica}

El enfoque que se ha esbozado revela por lo menos tres niveles ontológicos para el análisis metacientífico, a saber, las subestructuras, las poblaciones de modelos y los dominios temáticos. Consideramos que este enfoque se puede hacer extensivo a las prácticas instrumentales y a los contextos institucionales-sociales, aunque esto último sólo lo ofrecemos como posibilidad para la investigación futura, sin detenernos más en ello. De cualquier manera, el enfoque permite la introducción

tructuras, de tal suerte que regiones particulares de los grafos siempre están ocupadas por subestructuras de la misma clase de equivalencia. Proponemos el término de "nicho conceptual" para denotar estas regiones relacionadas de los grafos. 
de un marco evolucionista en el cual situar las dinámicas científicas; ésta, nos parece, es una de sus más destacadas virtudes. Permite tratar subestructuras -no necesariamente asociadas a modelos- que son relevantes en la gestación de dominios; además, constituye una buena herramienta para estudiar contextos locales, y da una imagen más real de la dinámica científica que aquella "fosilizada" en libros de texto.

Ahora bien, es menester explicitar algunos requisitos fundamentales para situar los modelos y dominios temáticos en un contexto evolucionista. Para discutir la evolución de las entidades $X$ es necesario, en primer término, proporcionar una caracterización adecuada de tales $X$ pero, además, es menester proporcionar también una descripción del tipo de cambio que sufre $X$, plantear uno o varios mecanismos de cambio y revelar uno o varios patrones del cambio. En el caso de la evolución "darwiniana” las modificaciones que sufren los organismos corresponden a pequeñas variaciones heredables de estructura, el mecanismo del cambio estriba en la selección natural y el patrón de estas modificaciones se vislumbra en filogenias arbóreas y gradualistas.

No es nuestra intención elevar algún principio selectivo a una metateoría de la dinámica científica, pero tampoco conviene soslayar sus posibilidades heurísticas. En biología, todas las teorías evolucionistas contemplan a la selección como un mecanismo evolutivo aunque, claro está, no en todas juega el papel central; en muchas, su función se limita a la "eliminación de monstruos". Dada la ubicuidad de la selección en las teorías sobre evolución orgánica, en los modelos de evolución conceptual resulta heurísticamente plausible recuperar una ontología mínima que permita la introducción de mecanismos selectivos. Como hemos mencionado, en principio podrían considerarse tres niveles de análisis: subestructura, modelo y dominio.

A continuación planteamos cómo nuestro enfoque se corresponde con los cuatro requisitos mencionados.

\subsection{Caracterización adecuada de las entidades que evolucionan}

Proponemos representar los modelos a modo de grafos, siendo cada grafo desglosable en un mosaico de subestructuras. Además, planteamos que tales grafos se agrupan en poblaciones y dominios temáticos, pudiendo ambos considerarse como unidad evolutiva. 


\subsection{El tipo de cambio que sufren las unidades evolutivas}

De manera no estadística, la caracterización del cambio conceptual se puede enunciar en términos del surgimiento o desaparición de las subestructuras que componen los modelos, o de los modelos mismos (dependiendo si hemos elegido a la población o al domino como unidad evolutiva). De manera estadística, la descripción del cambio conceptual se da en términos de modificaciones de las frecuencias relativas de las distintas subestructuras o modelos presentes en una población o en un dominio. Esta idea permite una caracterización cuantificable del cambio conceptual. El tiempo se concibe como una sucesión ordenada de instantes o lapsos y su escala depende del tipo de proceso bajo análisis (desde eventos que abarcan una o dos semanas hasta siglos); puede incluso plantearse una noción de tiempo discreta o continua, dependiendo básicamente de si nos interesa o no introducir una función de cambio diferenciable.

Las adiciones, sustracciones y/o modificaciones de las subestructuras que componen a los modelos funcionan de modo semejante a las ligeras variaciones morfológicas en la evolución biológica (mutaciones). La comunicación científica permite la transmisión cultural de estos ensambles conceptuales (heredabilidad).

\subsection{Los mecanismos del cambio científico}

Los mecanismos del cambio científico son múltiples y en ocasiones están imbricados o secuenciados. Algunos de ellos son:

(i) La creación intelectual y el surgimiento de auténticas novedades. Tanto estructuras completas, verbigracia la teoría de la relatividad, como conceptos aislados, por ejemplo, el de probabilidad. Lo importante a destacar aquí es que pareciera no haber antecedentes de tales novedades.

(ii) La modificación de modelos establecidos (por ejemplo todas las especializaciones de una red teórica o la incorporación de una variante en una población de modelos). Somos conscientes de que la diferencia entre novedades y modificaciones es borrosa, pero queremos mantener la distinción a fin de revelar la importancia de la variación dirigida como mecanismo distinto a la creación. 
(iii) La fusión teórica. Modelos y/o subestructuras de dos o más dominios y/o poblaciones distintas pueden sufrir un sincretismo y dar lugar a un nuevo dominio y/o modelo, tal es el caso de la llamada síntesis moderna (neodarwinismo) en la biología, producto de la fusión de la teoría cromosómica de la herencia mendeliana y la teoría de evolución por selección natural.

(iv) La selección de determinadas variantes de una población de modelos. Respecto a ésta adelantamos algunas ideas generales. Cuando los usuarios de las teorías privilegian un modelo sobre otro, entra en operación un criterio selectivo. Que sea pautado por las nociones de verdad, evidencia, parsimonia, adecuación empírica, etcétera, que los científicos involucrados en el tema hayan establecido por voluntad propia, o que sea producto de una coerción institucional, no le quita el carácter de selectivo, aunque sí pone de relieve la existencia de modalidades de selección.

La selección relaciona el valor de "satisfacción" de un tipo de modelo con su tasa de crecimiento respecto a un criterio previo (número de citas en la literatura, importancia de los problemas que se resuelven con él, etcétera, i.e. lo que podríamos denominar su "peso específico"). Si en determinado contexto y tiempo un cierto tipo de modelo posee un grado de satisfacción mayor que otro, entonces también poseerá un mayor índice de crecimiento.

Los contextos intelectuales son el análogo de los ambientes biológicos, su descripción se da en términos de intereses, valores, criterios, normas y demás variables. Un contexto intelectual se presenta de múltiples maneras; en primer lugar, en cuerpos humanos, pues de alguna manera nuestros modelos y nuestros intereses, valores, criterios, normas o reglas, coexisten en nosotros, pero también se pueden asociar contextos intelectuales a los grupos de investigación, a las instituciones, a los colectivos sociales, y en general a cualquier agente social.

(v) La deriva cultural. Al igual que la deriva génica, este mecanismo consiste en lo que podríamos llamar "errores de muestreo". Dentro de una población, tan sólo una porción, "aleatoriamente” escogida, trasmitirá su legado (social o genético) 
a las siguientes generaciones. Por ejemplo, ciertos cataclismos sociales eliminan científicos $-\mathrm{y}$, junto con ellos, sus ideas- por cuestiones ajenas a la propia ciencia. Lavoisier fue ejecutado por la Revolución Francesa y la Segunda Guerra Mundial eliminó de manera ciega a cientos de investigadores. De no haber ocurrido estos cataclismos muy probablemente la historia científica sería distinta. Este efecto es especialmente notorio en los países subdesarrollados pues, al no poseer tradiciones de investigación propias, son susceptibles a los efectos un tanto aleatorios de la migración de los hombres de ciencia. ${ }^{15}$ Los efectos de la deriva cultural también se manifiestan durante aquellos sucesos en los que científicos de una determinada disciplina orientan su atención sobre problemas de otra disciplina, e importan en el proceso una serie de conceptos, métodos e instrumentos que son más avalados por su pertenencia a la tradición anterior que por su adecuación al nuevo contexto.

\subsection{Los patrones evolutivos}

Un patrón evolutivo depende, en buena medida, del o los mecanismos evolutivos en acción, y conviene añadir que un mismo mecanismo (verbigracia la selección) actuando en niveles distintos genera patrones distintos.

Los posibles patrones de evolución son numerosos y diversos. Todas las posibilidades lógicas deben considerarse, no es posible descartar a priori ninguna; tan sólo por citar dos ejemplos, junto a un patrón de desarrollo acumulacionista por inclusión, subsunción o fusión de poblaciones de modelos, y sin extinción (la visión de la Concepción Heredada), puede ubicarse un patrón de cambio "saltacionista", donde se presenten grandes periodos en los que casi no ocurran cambios y la génesis de poblaciones de modelos sea un fenómeno raro y repentino (la visión kuhniana). Cual sea el caso entre ambos patrones es una cuestión histórica.

A continuación se presenta un grafo hipotípico que muestra el comportamiento de poblaciones en diversos dominios a lo largo del tiempo.

${ }^{15}$ La historia de la ciencia mexicana es una u otra antes y después de la influencia del exilio español 
La siguiente figura ilustra sinópticamente la evolución de dominios y de teorías dentro de los mismos. La abscisa representa la diversidad, y la ordenada el tiempo, los modelos individuales (grafos) se representan mediante líneas verticales, las distintas poblaciones son conjuntos cercanos de éstas en un mismo color y tono, las líneas horizontales representan relaciones de especialización entre modelos, ${ }^{16}$ las líneas gruesas demarcan dominios, la secuencia de cuadros de colores que aquí hemos indicado sólo bajo algunas líneas representa la composición de tales modelos en términos de subestructuras (cada cuadro de color representa una subestructura), y la secuencia de colores indica su posición en la abcisa.
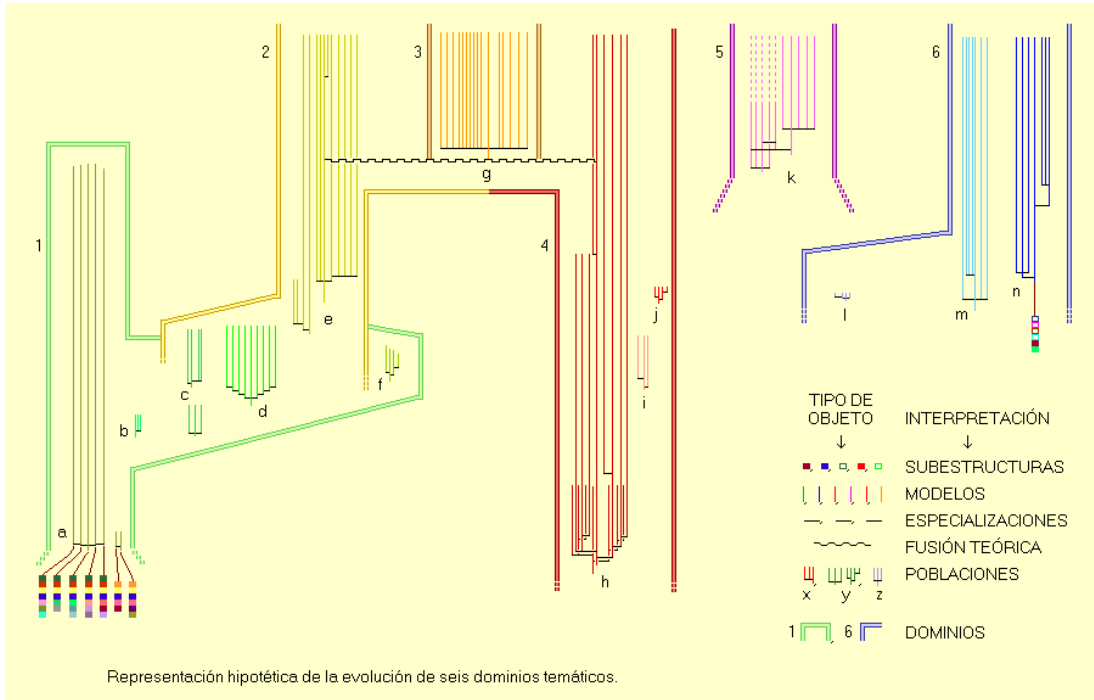

Figura 6: Representación de la evolución de dominios.

Respecto a las poblaciones nos permitimos destacar los siguientes casos: c representa la extinción y posterior renacimiento de una población (verbigracia, la teoría de la endosimbiosis serial sobre el origen de los organelos eucarióticos que, si bien se había propuesto en 1940, fue totalmente desconsiderada por la respectiva comunidad de biólogos, para reaparecer con vigor -aunque ligeramente modificada- en las propuestas de Margulis y Gupta de los años setenta del siglo pasado);

${ }^{16}$ Nótese que algunas poblaciones (a, e y h) están conformadas por mas de una red. 
d representa un cono de diversidad creciente, de acuerdo a los cánones del desarrollo científico de la Concepción Heredada; k pone de relieve la asimilación de una red previa por una nueva, lo que trae como consecuencia que la vieja, si todavía se mantiene, se restrinja a un ámbito meramente educativo (líneas punteadas); j y l podrían representar la deriva cultural por extinción de los postulantes.

En la figura se observan los siguientes patrones: (1) la posibilidad de que determinadas subestructuras sean compartidas por más de una población, o incluso que determinadas subestructuras salten de un dominio a otro (recuadros bajo a y n); (2) discontinuidad de las poblaciones dentro de un dominio y continuidad de dominios; ${ }^{17}$ (3) ocurrencia tanto de hegemonía de una población ( $\mathrm{g}$ y k) como de diversidad de poblaciones (a-f, h-j, l-n) dentro de un dominio; (4) surgimiento de novo de nuevos dominios (verde, rojo, morado y azul); (5) nacimiento de un dominio dentro de otro (amarillo dentro de verde) o bifurcación de dominios; (6) fusión de dominios (rojo y amarillo que engendran naranja); 7) extinción de dominios (verde); (8) en el caso del surgimiento de nuevos dominios por fusión de dominios previos, los límites de estos últimos se tornan difusos en el territorio de intersección; (9) los diferentes límites de un dominio pueden surgir de manera no simultánea (amarillo), pues si bien se puede dar la diferenciación a ciertos territorios conceptuales, también puede existir ambigüedad respecto a otros. Tanto surgimiento como extinción pueden ser más o menos continuos o abruptos.

Como se ve, no hemos representado rupturas de dominios à la Kuhn ni discontinuidad entre dominios. Un nuevo domino surge dentro de otro, de novo o por fusión de dominios, ${ }^{18}$ pero esto representa tan sólo una preferencia personal de los autores y no una limitación metodológica de la concepción grafo-modélica.

\footnotetext{
${ }^{17}$ Nos parece que la diferencia entre población y dominio subsana muchas de las ambigüedades asociadas al uso del término 'paradigma'.

${ }^{18}$ Por ejemplo, el domino de la herencia surge dentro de otros dominios: la teratología, la medicina, la hibridación (criadores), la citología. La herencia tiene raíces en éstos que posteriormente se fusionan entre sí para dar lugar a un nuevo tronco: el domino de la herencia; dentro de éste nacen las genéticas.
} 


\section{Conclusiones}

La representación gráfica de los modelos teóricos y el reconocimiento de poblaciones de modelos y dominios temáticos constituyen herramientas útiles para la metaciencia. De alguna manera, el enfoque aquí expuesto está en la búsqueda de una representación "dialógica" de la dinámica científica. El objetivo es perfeccionar una especie de so-

porte conceptual que sirva para vislumbrar las propuestas y polémicas científicas, en términos de transacciones estructurales de sus modelos. El énfasis en la visualización no es mera retórica; el presente trabajo y la línea de investigación que de él se desprende privilegian la imagen como auxiliar del entendimiento (somos primates: visualizamos, imaginamos, figuramos, etc.).

A pesar de que el enfoque poblacional aquí esbozado incide sobre el aspecto conceptual del quehacer científico, no creemos que se limite a él. Cabe la posibilidad, aún no trabajada, de que se pueda extender al terreno de la práctica instrumental. Los puntos ya no representarían entidades conceptuales, ni las flechas relaciones de inferencia, sino más bien objetos empíricos y relaciones de actuación. Quizá aquí nuestros modelos ya no sean grafos cerrados, preferiblemente conmutativos, sino algo parecido a árboles. Incluso, al representar un determinado tipo de instrumento - por ejemplo un osciloscopio- quizá nos veamos confrontados con algo así como conglomerados de grafos teóricos -esto es, las teorías que se concretizan en el osciloscopio- y grafos instrumentales, conectados entre sí por relaciones que pongan de relieve vínculos pragmáticos-inventivos.

También cabe la posibilidad de extender el enfoque poblacional a contextos institucionales-sociales, aunque esto se ve todavía más remoto De cualquier manera, no es descabellada una representación estructural de tales contextos y seguramente la noción de que se pueden desglosar en subestructuras, y éstas a su vez en subestructuras más finas, sirva para tal propósito.

En fin, estas dos extensiones del enfoque modelo-poblacional son apenas una promesa y, sin duda, se enfrentarán a diversos problemas de aplicación; no obstante, la idea nos parece sumamente atractiva. 


\section{Bibliografía}

Balzer, W., C.U. Moulines, y J.D. Sneed, 1987, An Architectonic for Science. The Structuralist Program, D. Reidel Publishing Company, Dordrecht.

Barwise, J. y J. Seligman, 1997, Information Flow. The Logic of Distributed Systems, Cambridge University Press, Cambridge.

Bateson, W., 1902, Mendel's Principals of Heredity: A Defense, London, Cambridge University Press. En http://www.esp.org./foundations/genetics/classical/browse/.

Casanueva, M., 2003, Mendeliana, Miguel Angel Porrúa/Universidad Autónoma Metropolitana, México.

Darwin, C., 1883, The Variation of Animals and Plants under Domestication, 2da. edición, revisada (2 volúmenes), D. Appleton, Nueva York. En http://www.esp.org./foundations/genetics/classical/browse/.

Díez, J. A. y C.U. Moulines, 1999, Fundamentos de filosofía de la ciencia. 2da. edición, Ariel, Barcelona.

Fisher, R.A., 1918, "The Correlation Between Relatives on the Supposition of Mendelian Inheritance", Transactions of the Royal Society of Edinburgh, vol. 52, pp. 399-433.

Galton, F., 1889, Natural Inheritance, Macmillan, Londres. En http://www.esp.org./foundations/genetics/classical/browse/.

Hardy, G. H., 1908, "Mendelian Proportions in a Mixed Population", Science, no. 28, pp. 49-50. En http://www.esp.org./foundations/genetics/classical/browse/.

Mayr, E., 1982, The Growth of Biological Thought, Harvard University Press, Cambridge, Mass.

Mendel, G., 1865, "Experiments In Plant Hybridization”. En http://www.esp.org./foundations/gene-tics/classical/browse/.

Poincaré, H., 1963, Ciencia y método, Espasa-Calpe, Madrid.

Sutton, W. S., 1903, "The Chromosome in Heredity", Biological Bulletin, vol. 4, pp. 231-251. En http://www.esp.org./foundations/genetics/classical/browse/.

De Vries, H., 1910, Intracellular Pangenesis, Open Court Publishing, Chicago. En http://www.esp.org./foundations/genetics/classical/browse/.

Weismann, A., 1893, The Germplasm, Charles Shribner's and Sons, Nueva York. En http://www.esp.org./foundations/genetics/classical/browse/.

Yule, G.U., 1902, "Mendel's Laws and Their Probable Relations to Intra-Racial Heredity”, New Phytologist, vol. 1, no. 9, pp. 193-207.

Recibido el 8 de septiembre de 2011 Aceptado el 30 de noviembre de 2011 